\title{
USOS DEL TIEMPO DE MUJERES Y HOMBRES EN LA GOMERA: UN ESTUDIO DE CASO
}

\author{
USES OF TIME OF WOMEN AND MEN IN LA GOMERA ISLAND: A CASE \\ STUDY
}

\author{
Josué Gutiérrez Barroso \\ Universidad de La Laguna (España) \\ igb 84@hotmail.com
}

Recibido/Received: 23/03/2013

Aceptado/Accepted: 30/06/2013

\section{RESUMEN}

En esta investigación, financiada por el Excmo. Cabildo Insular de La Gomera, se analizarán las diferencias y semejanzas de mujeres y hombres de la Isla de La Gomera a través del estudio de los usos del tiempo. La importancia del estudio viene dada por el análisis de una zona con peculiaridades demográficas que influyen en el plano de la igualdad de género y la posibilidad de establecer diferencias con el resto del territorio español. Para ello, se aplicó la técnica de la encuesta a los hombres y mujeres habitantes de La Gomera, obteniendo unos resultados interesantes a la hora de observar las diferencias entre ambos sexos según los diferentes usos del tiempo estudiados (tiempo al día dedicado a necesidades personales, trabajo doméstico, cuidado de la familia, mantenimiento del hogar, tareas del hogar fuera del mismo, tiempo libre y trabajo remunerado). Además, se compararon los resultados con otro estudio similar realizado por el Instituto de la Mujer.

\section{PALABRAS CLAVE}

Tiempo social, hombre, mujer, igualdad.

\section{SUMARIO}

1. Antecedentes y justificación de la investigación. 2. Metodología. 3. Análisis de los usos del tiempo según sexo y edad. 4. Análisis de la diferencia de usos del tiempo según el sexo y el resto de variables de control. 5. Comparativa nacional-insular. 6. Conclusiones. Bibliografía.

\begin{abstract}
In this research, funded by the Cabildo Insular de La Gomera, analyzes differences and similarities of women and men on La Gomera Island studying the uses of time. The importance of the study is given by the analysis of an area with demographic characteristics that influence the level of gender equality and the possibility of differences with the rest of the Spain. To do this, we applied the technique of the survey to the men and women who live in La Gomera, getting interesting results when observing the differences between gender and uses of time (day time devoted to personal needs, housework, family care, home maintenance, household chores outside it, leisure, and work). Furthermore, we compared the results with a similar study which have been done by the Institute for Women.
\end{abstract}

\section{KEYWORDS}

Social time, man, woman, time, equality. 


\section{CONTENTS}

1. Background and rationale of research. 2. Methodology. 3. Analysis of the use of time by sex and age. 4. Analysis of the use of time difference by sex and other control variables. 5. National-insular comparative. 6. Conclusions. References.

\section{ANTECEDENTES Y JUSTIFICACIÓN DE LA INVESTIGACIÓN}

Como antecedente teórico más lejano al objetivo de esta investigación, según destaca Álvaro Page (1996), encontramos en el ámbito español el estudio de Izquierdo et al. (1988). En él, estudian las diferencias de los usos del tiempo entre mujeres y hombres a través de tres grandes grupos: el trabajo doméstico, el trabajo remunerado y el tiempo para el resto de actividades. En dichas esferas los autores detectan diferencias considerables en los usos del tiempo, pues detectan que el sexo es la variable más importante en la distribución del tiempo entre mujeres y hombres. Ya en 1990, Ramos Torres publica su obra Cronos dividido. Uso del tiempo y desigualdades entre mujeres y hombres en España (Ramos, 1990), donde agrupa las actividades en cuatro grandes bloques: trabajo profesional y académico, trabajo doméstico y familiar, necesidades esenciales y tiempo libre, llegando también a establecer diferencias significativas entre hombres y mujeres.

A partir de los años 90 las investigaciones sobre los usos del tiempo adquieren una mayor magnitud. Así, el CSIC realiza a partir de ese momento investigaciones de este tipo tanto a nivel nacional como regional (1990, 1995, 1998, 2003, 2005 y 2008); al igual que el CIRES (1991, 1996) y el CIS $(2000,2008)$. Por otro lado, el INE también ha realizado estudios sobre los usos del tiempo bastante rigurosos, cuya última publicación es el realizado en 2011. Sin embargo, el antecedente más inmediato que se asemeja al estudio que se realizó en La Gomera es el titulado Usos del tiempo, estereotipos, valores y actitudes (De la Fuente, 2007), que trata de constatar las posibles diferencias en cuanto al universo valorativo y actitudinal entre mujeres y hombres en España. Por ello, al tener un objetivo y una metodología similar al del presente proyecto, dicho estudio inspiró la mayor parte de la distribución sobre los usos del tiempo a investigar en la Isla de La Gomera, todo ello con el fin de obtener resultados relativamente comparables.

Esta investigación, financiada por el Excmo. Cabildo Insular de La Gomera, se dispone a determinar las diferencias en cuanto a usos del tiempo entre mujeres y hombres en la Isla de La Gomera y establecer la comparativa del estudio insular con los datos recogidos a nivel nacional. El análisis de este enclave insular surge partiendo de la hipótesis de que, dadas las peculiaridades demográficas de La Gomera, sería interesante analizar la situación de las mujeres y hombres en la isla sobretodo relacionado con la desigualdad en los usos del tiempo entre ambos sexos.

La Gomera es una Isla perteneciente a la provincia de Santa Cruz de Tenerife formada por seis municipios cuya población asciende a 22.769 habitantes según la Explotación Estadística del Padrón a fecha 1/1/2009 (INE). Este enclave posee un índice de envejecimiento bastante elevado (18,21\%) y un índice de juventud extremadamente bajo (12,35\%), lo cual le hace ser un territorio especialmente complicado a la hora de desarrollarse social y económicamente. Por ello, y dado estos condicionantes, resulta interesante observar cómo éstos afectan a la vida diaria de los hombres y mujeres, y así tratar de vislumbrar las posibles diferencias entre la Isla y el resto del territorio nacional. En principio, cabría esperar que dado su carácter rural, en La Gomera exista una desigualdad que en muchos casos sea superior a la existente a 
nivel nacional en lo que a usos del tiempo entre hombres y mujeres se refiere, por lo que esta será nuestra hipótesis de investigación.

\section{METODOLOGÍA}

La metodología utilizada en esta investigación es de tipo cuantitativa, en concreto se usó la técnica de la encuesta. Sin embargo, es necesario realizar varias aclaraciones. En primer lugar, las entrevistas se hicieron en los hogares a través de un cuestionario con preguntas que realizaba el encuestador y contestaba el encuestado. De este modo, se optó por esa forma (empleada por De la Fuente Sánchez, 2007) en vez de por el diario de actividades (empleado por el INE) debido a necesidades de financiación y de tiempo de la investigación. Además, hay que tener en cuenta que se han seguido las recomendaciones acerca de las encuestas sobre usos del tiempo y sus dificultades comparativas recogidas por Durán (2009) y Raldúa (2001), en cuanto a la selección y clasificación de actividades.

Por otro lado, no se contabilizan las horas dedicadas al sueño y es necesario aclarar que, como en el estudio de De la Fuente Sánchez (2007), en esta investigación el parámetro a estudiar es el valor medio respecto a la muestra, independiente de que se dedique, o no, tiempo a una actividad concreta. De esta forma, algunas de las cifras expuestas parecen más bajas en principio si no se tiene en cuenta que van referidas al conjunto de la población entrevistada. Aparte de lo anterior, al analizar los usos del tiempo conviene recordar que el tiempo dedicado a todas las actividades puede sumar más de 24 horas en un día, lo cual viene dado porque se admitió la posibilidad de realizar varias actividades de forma simultánea.

Partiendo de un universo poblacional de 19.197 habitantes mayores de 18 años, se previó, a partir de un nivel de confianza del $95 \%$ y un error del ${ }^{+}-5 \%$, una muestra de 376 individuos a los que se les aplicó dicha encuesta. Sin embargo, debido a que la temporalidad lo permitió, la cantidad de encuestas realizadas se aumentó respecto a lo que se había previsto en el proyecto, siendo la muestra final de 425 individuos según se expone en la tabla 1 . Dicha encuesta se realizó entre los meses de mayo a julio de 2009.

TABLA 1: Muestra final para el estudio. La Gomera 2009

\begin{tabular}{|c|c|c|c|c|c|c|c|c|}
\hline \multicolumn{2}{|c|}{ Muestra total } & \multicolumn{2}{c|}{18 a 45 años } & \multicolumn{2}{c|}{46 a 64 años } & \multicolumn{2}{c|}{65 y más } & \multirow{2}{*}{ Total } \\
\cline { 1 - 7 } Hombres & Mujeres & Hombres & Mujeres & Hombres & Mujeres & Hombres & Mujeres & \\
\hline 213 & 212 & 102 & 122 & 65 & 52 & 46 & 38 & 425 \\
\hline
\end{tabular}

Fuente: Datos de Investigación

El muestreo fue estratificado por municipios (con el establecimiento de cuotas de sexo y edad en función de la población empadronada en cada entidad local) y, dentro del mismo, se distribuyó la muestra según los diferentes núcleos de población del municipio, pues se tuvo en cuenta la enorme diseminación poblacional en la Isla de La Gomera.

La selección de la muestra fue sistemática con arranque aleatorio. Por lo tanto, la selección de individuos fue aleatoria, pero siempre teniendo en cuenta, tal y como se afirmó anteriormente, las cuotas establecidas por sexo y edad en los diferentes núcleos de población de los municipios. Al tener todos los municipios una distribución por sexos en torno al $50 \%$, se intentó que la mitad de las entrevistas se realizaran a las mujeres y la otra mitad a los hombres (aplicado a cada tramo de edad). 
Cabe destacar que las entrevistas se hicieron tanto en hogares como en sitios de trabajo (bares, obras...), siempre siguiendo la ruta aleatoria de los encuestadores. La razón de escoger ambos lugares a la hora de aplicar la encuesta es que, al tener (por razones de disponibilidad del personal) encuestadores sólo de mañana, si no se asistía a los lugares de trabajo no se conseguía personas de entre 18 y 60 años puesto que la mayor parte de éstos en el municipio no se encontraba en los hogares. Aún así, se tuvo en cuenta que la población encuestada en cada entidad local estuviera empadronada en el propio municipio donde se realizaba la entrevista.

Las variables relativas a los usos del tiempo, incluidas en la investigación, son las siguientes:

1. Tiempo empleado al día para las Necesidades Personales (comer, asearse, levantarse, descansar...),

2. Tiempo empleado al día para el Trabajo Doméstico (barrer, limpiar el polvo, fregar, cocinar, lavar y tender la ropa, planchar...),

3. Tiempo empleado al día para el Cuidado de la Familia (cuidado de niños/as, ayuda en deberes a hijos, cuidado de mayores y discapacitados...),

4. Tiempo empleado al día para el Mantenimiento del Hogar y enseres comunes de la familia (cuidado de plantas, animales domésticos, del coche, de pequeñas reparaciones del hogar...),

5. Tiempo empleado al día para las Tareas del Hogar fuera del mismo (hacer la compra, visitas médicas, asistencia a reuniones de padres...),

6. Tiempo empleado al día para el Tiempo Libre (Charlas, caminar, leer, establecer relaciones sociales, viajar, actividades de entretenimiento, deporte...) y

7. Tiempo empleado al día para el Trabajo Remunerado.

\section{ANÁLISIS DE LOS USOS DEL TIEMPO SEGÚN EL SEXO Y LA EDAD DEL ENCUESTADO}

Tal y como se observa en la tabla 2, existen algunas tareas en las que los hombres y mujeres se diferencian, si bien hay otras cuya duración es muy similar para ambos sexos. Los datos nos muestran que, a priori y a falta de justificar estadísticamente, puede existir algún tipo de relación entre algunos usos del tiempo y el sexo del encuestado. De esta forma, en cuanto al trabajo doméstico, donde existe la diferencia entre sexos más significativa, las mujeres emplean casi tres veces y medio más de tiempo que los hombres, un dato a tener en cuenta a la hora de extraer conclusiones en materia de reparto de tareas en dicho ámbito

Una situación similar ocurre en cuanto al tiempo empleado para el cuidado de la familia, pues las mujeres emplean casi una hora más al día a este quehacer con respecto al otro sexo. También el sexo femenino emplea una mayor cantidad de tiempo para la realización de tareas del hogar fuera del mismo, aunque las diferencias son mucho menores. Por otro lado, los hombres emplean mucho más tiempo que las mujeres en dos ámbitos: el de tiempo libre y el del trabajo remunerado. Con respecto al segundo las diferencias son sustanciales, de casi una hora, lo cual es una muestra de una mayor tasa de población empleada masculina que femenina. A priori se puede concluir que el hecho de que los hombres empleen más tiempo en dichas cuestiones influye en que usen menos tiempo para el cuidado de la familia y el trabajo doméstico, feminizando, como ya se dijo anteriormente, estas últimas tareas. 
TABLA 2: Usos del tiempo al día según sexo del encuestado. La Gomera 2009.

\begin{tabular}{|c|c|c|c|c|c|c|c|}
\hline Sexo & $\begin{array}{l}\text { Tiempo } \\
\text { empleado } \\
\text { para } \\
\text { necesidades } \\
\text { personales } \\
\text { (comer, } \\
\text { aseo...) }\end{array}$ & $\begin{array}{l}\text { Tiempo } \\
\text { empleado } \\
\text { para el } \\
\text { trabajo } \\
\text { doméstico } \\
\text { (barrer, } \\
\text { limpiar } \\
\text { polvo, } \\
\text { cocinar, } \\
\text { lavar...) }\end{array}$ & $\begin{array}{l}\text { Tiempo } \\
\text { empleado } \\
\text { para el } \\
\text { cuidado de } \\
\text { la familia } \\
\text { (niños, } \\
\text { mayores, } \\
\text { ayuda en } \\
\text { deberes) }\end{array}$ & $\begin{array}{l}\text { Tiempo } \\
\text { empleado para } \\
\text { el } \\
\text { mantenimiento } \\
\text { (del coche, } \\
\text { plantas, } \\
\text { animales...) }\end{array}$ & $\begin{array}{l}\text { Tiempo } \\
\text { empleado } \\
\text { para la } \\
\text { realización } \\
\text { de tareas } \\
\text { del hogar } \\
\text { fuera del } \\
\text { mismo } \\
\text { (compras, } \\
\text { visitas } \\
\text { médicas,...) }\end{array}$ & $\begin{array}{l}\text { Tiempo } \\
\text { empleado } \\
\text { para tiempo } \\
\text { libre } \\
\text { (charlar, } \\
\text { lectura, } \\
\text { relaciones } \\
\text { sociales, } \\
\text { viajes, } \\
\text { entretenimi } \\
\text { ento...) }\end{array}$ & $\begin{array}{l}\text { Tiempo } \\
\text { empleado } \\
\text { para trabajo } \\
\text { remunerado }\end{array}$ \\
\hline Hombre & $\begin{array}{l}1 \text { hora y } 55 \\
\text { minutos }\end{array}$ & 44 minutos & 44 minutos & 58 minutos & 43 minutos & $\begin{array}{l}1 \text { hora y } 46 \\
\text { minutos }\end{array}$ & $\begin{array}{l}5 \text { horas y } 57 \\
\text { minutos }\end{array}$ \\
\hline Mujer & $\begin{array}{l}2 \text { horas y } 2 \\
\text { minutos }\end{array}$ & $\begin{array}{l}2 \text { horas y } \\
33 \text { minutos }\end{array}$ & $\begin{array}{l}1 \text { hora y } 33 \\
\text { minutos }\end{array}$ & $\begin{array}{l}1 \text { hora y } 2 \\
\text { minutos }\end{array}$ & $\begin{array}{l}1 \text { hora y } 24 \\
\text { minutos }\end{array}$ & $\begin{array}{l}1 \text { hora y } 24 \\
\text { minutos }\end{array}$ & $\begin{array}{l}4 \text { horas y } 46 \\
\text { minutos }\end{array}$ \\
\hline TOTAL & $\begin{array}{l}1 \text { hora y } 59 \\
\text { minutos }\end{array}$ & $\begin{array}{l}1 \text { hora y } 38 \\
\text { minutos }\end{array}$ & $\begin{array}{l}1 \text { hora y } 8 \\
\text { minutos }\end{array}$ & 59 minutos & $\begin{array}{l}1 \text { hora y } 2 \\
\text { minutos }\end{array}$ & $\begin{array}{l}1 \text { hora y } 36 \\
\text { minutos }\end{array}$ & $\begin{array}{l}5 \text { horas y } 22 \\
\text { minutos }\end{array}$ \\
\hline
\end{tabular}

Fuente: Datos de Investigación

Respecto a la media de usos del tiempo empleado y la relación con el sexo y la edad (tabla 3), los resultados también resultan interesantes a la hora de mostrar la realidad social de la igualdad de género en la Isla de La Gomera. En cuanto a las necesidades personales, donde menos tiempo emplean son en los intervalos de 30 a 64 años, tanto hombres como mujeres, siendo el primero (18 a 29) y el último (65 y más) en los que más tiempo invierten. Por otro lado, en cuanto al trabajo doméstico, y tal y como se observó en la tabla $\mathrm{V}$, los hombres emplean menos tiempo que las mujeres en todos los intervalos, llegando a ser la diferencia mayor en el de 65 y más años.

Respecto al tiempo empleado para el mantenimiento del hogar, ocurre algo similar que con el tiempo libre: emplean los hombres y las mujeres tiempos similares en todos los intervalos menos en uno. Los encuestados de sexo masculino de entre 65 y más años emplean casi una hora más en su tiempo libre que las mujeres, siendo la mayor diferencia existente en dicho ámbito. En el cuidado de la familia, los hombres emplean menos tiempo que las mujeres en todos los intervalos de edad, algo que también ocurre en cuanto al desarrollo de tareas fuera del hogar. A la inversa ocurre si tenemos en cuenta el trabajo remunerado, algo en lo que los hombres invierten más tiempo debido a la razón expuesta en el apartado anterior.

Estudiando los grupos de edad, se observa que los jóvenes tienen una distribución similar a la población mayor de 65 años en lo que se refiere al trabajo doméstico, por lo que se intuye que se siguen repitiendo ciertos patrones de desigualdad en cuanto a las tareas del hogar. Además, dicha desigualdad también se observa en el hecho de que los hombres jóvenes emplean casi una hora más que las mujeres en el trabajo remunerado. En cuanto a los adultos (de 30 a 64), es donde, en todos los ámbitos, más igualdad puede existir, excluyendo el ámbito del trabajo doméstico y el cuidado de la familia, si bien existen ciertas diferencias que bien podrían ser indicios de desigualdad. Por último, la población mayor de 65 años expresa las mayores diferencias por sexo en lo que se refiere al trabajo doméstico, si bien es llamativo que existe casi la misma que el sector juvenil, como bien se dijo anteriormente. Además, es lógico que la población anciana disponga de un mayor tiempo libre y un espacio temporal más amplio para el desarrollo de sus actividades de ocio con respecto a otros grupos 
de edad, pues la mayoría están jubilados. Obviamente, por esa misma razón, el trabajo remunerado es un ámbito poco significativo respecto a este sector etario.

TABLA 3: Usos del tiempo al día según edad y sexo del encuestado. La Gomera 2009

\begin{tabular}{|c|c|c|c|c|c|c|}
\hline Usos del tiempo & Sexo & 18 a 29 & 30 a 44 & 45 a 64 & 65 y más & TOTAL \\
\hline \multirow{2}{*}{$\begin{array}{l}\text { Tiempo Empleado } \\
\text { para Necesidades } \\
\text { personales (comer, } \\
\text { aseo...) }\end{array}$} & Hombres & $\begin{array}{l}2 \text { horas y } 22 \\
\text { minutos }\end{array}$ & $\begin{array}{l}1 \text { hora y } 45 \\
\text { minutos }\end{array}$ & $\begin{array}{l}1 \text { hora y } 49 \\
\text { minutos }\end{array}$ & $\begin{array}{l}1 \text { hora y } 55 \\
\text { minutos }\end{array}$ & $\begin{array}{l}1 \text { hora y } 55 \\
\text { minutos }\end{array}$ \\
\hline & Mujeres & $\begin{array}{l}2 \text { horas y } 37 \\
\text { minutos }\end{array}$ & $\begin{array}{l}1 \text { hora y } 53 \\
\text { minutos }\end{array}$ & $\begin{array}{l}1 \text { hora y } 43 \\
\text { minutos }\end{array}$ & $\begin{array}{l}2 \text { horas y } 5 \\
\text { minutos }\end{array}$ & $\begin{array}{l}2 \text { horas y } 2 \\
\text { minutos }\end{array}$ \\
\hline \multirow{2}{*}{$\begin{array}{l}\text { Tiempo Empleado } \\
\text { para el Trabajo } \\
\text { Doméstico (barrer, } \\
\text { limpiar polvo, cocinar, } \\
\text { lavar...) }\end{array}$} & Hombres & 43 minutos & 1 hora & 48 minutos & 19 minutos & 45 minutos \\
\hline & Mujeres & $\begin{array}{l}2 \text { horas y } 6 \\
\text { minutos }\end{array}$ & $\begin{array}{l}2 \text { horas y } 36 \\
\text { minutos }\end{array}$ & $\begin{array}{l}2 \text { horas y } 45 \\
\text { minutos }\end{array}$ & $\begin{array}{l}2 \text { horas y } 44 \\
\text { minutos }\end{array}$ & $\begin{array}{l}2 \text { horas y } 33 \\
\text { minutos }\end{array}$ \\
\hline \multirow{2}{*}{$\begin{array}{l}\text { Tiempo Empleado } \\
\text { para el Cuidado de la } \\
\text { familia (niños, } \\
\text { mayores, ayuda en } \\
\text { deberes) }\end{array}$} & Hombres & $38 \mathrm{mi}$ & $\begin{array}{l}1 \text { hora y } 15 \\
\text { minutos }\end{array}$ & 41 minutos & 12 minutos & 44 minutos \\
\hline & Mujeres & $\begin{array}{l}1 \text { hora y } 32 \\
\text { minutos }\end{array}$ & $\begin{array}{l}2 \text { horas y } 7 \\
\text { minutos }\end{array}$ & $\begin{array}{l}1 \text { hora y } 32 \\
\text { minutos }\end{array}$ & 34 minutos & $\begin{array}{l}1 \text { hora y } 33 \\
\text { minutos }\end{array}$ \\
\hline \multirow{2}{*}{$\begin{array}{l}\text { Tiempo Empleado } \\
\text { para el Mantenimiento } \\
\text { (del coche, plantas, } \\
\text { animales...) }\end{array}$} & Hombres & 40 minutos & 54 minutos & 59 minutos & $\begin{array}{l}1 \text { hora y } 17 \\
\text { minutos }\end{array}$ & 58 minutos \\
\hline & Mujeres & 47 minutos & 55 minutos & $\begin{array}{l}1 \text { hora y } 26 \\
\text { minutos }\end{array}$ & 57 minutos & $\begin{array}{l}1 \text { hora y } 2 \\
\text { minutos }\end{array}$ \\
\hline \multirow{2}{*}{$\begin{array}{l}\text { Tiempo Empleado } \\
\text { para la realización de } \\
\text { Tareas del hogar fuera } \\
\text { del mismo (compras, } \\
\text { visitas médicas,...) }\end{array}$} & Hombres & 378 minutos & 50 minutos & 31 minutos & 56 minutos & 43 minutos \\
\hline & Mujeres & $\begin{array}{l}1 \text { hora y } 38 \\
\text { minutos }\end{array}$ & $\begin{array}{l}1 \text { hora y } 10 \\
\text { minutos }\end{array}$ & $\begin{array}{l}1 \text { hora y } 23 \\
\text { minutos }\end{array}$ & $\begin{array}{l}1 \text { hora y } 22 \\
\text { minutos }\end{array}$ & $\begin{array}{l}1 \text { hora y } 24 \\
\text { minutos }\end{array}$ \\
\hline \multirow{2}{*}{$\begin{array}{l}\text { Tiempo Empleado } \\
\text { para Tiempo Libre: } \\
\text { Charlar, lectura, } \\
\text { relaciones sociales, } \\
\text { viajes, entretenimiento }\end{array}$} & Hombres & $\begin{array}{l}1 \text { hora y } 51 \\
\text { minutos }\end{array}$ & $\begin{array}{l}1 \text { hora y } 41 \\
\text { minutos }\end{array}$ & $\begin{array}{l}1 \text { hora y } 31 \\
\text { minutos }\end{array}$ & $\begin{array}{l}2 \text { horas y } 14 \\
\text { minutos }\end{array}$ & $\begin{array}{l}1 \text { hora y } 46 \\
\text { minutos }\end{array}$ \\
\hline & Mujeres & $\begin{array}{l}1 \text { hora y } 59 \\
\text { minutos }\end{array}$ & $\begin{array}{l}1 \text { hora y } 12 \\
\text { minutos }\end{array}$ & $\begin{array}{l}1 \text { hora y } 17 \\
\text { minutos }\end{array}$ & $\begin{array}{l}1 \text { hora y } 15 \\
\text { minutos }\end{array}$ & $\begin{array}{l}1 \text { hora y } 24 \\
\text { minutos }\end{array}$ \\
\hline \multirow{2}{*}{$\begin{array}{l}\text { Tiempo Empleado } \\
\text { para Trabajo } \\
\text { Remunerado }\end{array}$} & Hombres & $\begin{array}{l}5 \text { horas y } 44 \\
\text { minutos }\end{array}$ & $\begin{array}{l}8 \text { horas y } 2 \\
\text { minutos }\end{array}$ & $\begin{array}{l}7 \text { horas y } 45 \\
\text { minutos }\end{array}$ & 30 minutos & $\begin{array}{l}5 \text { horas y } 57 \\
\text { minutos }\end{array}$ \\
\hline & Mujeres & $\begin{array}{l}4 \text { horas y } 33 \\
\text { minutos }\end{array}$ & $\begin{array}{l}6 \text { horas y } 54 \\
\text { minutos }\end{array}$ & $\begin{array}{l}5 \text { horas y } 11 \\
\text { minutos }\end{array}$ & 28 minutos & $\begin{array}{l}4 \text { horas y } 46 \\
\text { minutos }\end{array}$ \\
\hline
\end{tabular}

Fuente: Datos de Investigación

\section{ANÁLISIS DE LA DIFERENCIA DEL USO DEL TIEMPO SEGÚN SEXO Y EL RESTO DE VARIABLES DE CONTROL.}

En el bloque anterior se analizaron las diferencias en los usos del tiempo según las variables sexo y edad. Sin embargo, es necesario realizar un análisis estadístico que permita medir la influencia, además del sexo, de otras variables denominadas "de control" (independientes). Las variables de control del modelo que se ha realizado y que se aplicarán a los diferentes usos del tiempo (variables dependientes) son las siguientes: sexo, edad, estado civil, nivel de estudios y miembros en el hogar menores de 16 años. Para la tarea anterior se 
realizará un análisis de covarianza, todo ello con el objetivo de determinar que variables influyen más en las variaciones de las variables relacionadas con los usos del tiempo (variables dependientes). Con el objetivo de lograr una interpretación más adecuada, desde el momento en el que se detecte, mediante el análisis de la covarianza, que una variable de control es significativa (significatividad menor que 0,05 ), se procederá a mostrar la tabla de contingencia donde aparezca dicha variable y el uso del tiempo que se esté analizando.

Tal y como se observa en la tabla 4, el tiempo dedicado a tareas relacionadas con las necesidades personales depende, de entre todas las variables incluidas en el modelo, del número de miembros menores de 16 años incluidos en el hogar, pues la significatividad de la misma es menor que 0,05 .

TABLA 4: Variable "tiempo dedicado a necesidades personales" según fuente de variación. La Gomera 2009.

\begin{tabular}{|l|l|l|}
\hline Fuente de Variación & F & Significatividad \\
\hline Edad & 1,595 & 0,208 \\
\hline Miembros en el hogar menores de 16 años & 6,849 & 0,009 \\
\hline Sexo & 1,680 & 0,196 \\
\hline Estado Civil & 0,385 & 0,764 \\
\hline Nivel de estudios terminados & 0,480 & 0,791 \\
\hline Interacción Sexo * Estado Civil & 1,122 & 0,341 \\
\hline Interacción Sexo * Nivel de estudios terminados & 0,402 & 0,847 \\
\hline Interacción Estado Civil * Nivel de estudios terminados & 1,885 & 0,054 \\
\hline Interacción Sexo * Nivel de estudios terminados * Estado Civil & 0,903 & 0,505 \\
\hline Varianza explicada por el modelo & $69,00 \%$ & \\
\hline
\end{tabular}

Fuente: Datos de Investigación

De esta forma, al comparar ambas variables en una tabla de contingencia (tabla 5), ésta nos muestra que a medida que el número de miembros del hogar menores de 16 años aumenta, menor es el tiempo empleado para dicha cuestión.

TABLA 5: Número de miembros del hogar menores de 16 años y tiempo empleado para necesidades personales. La Gomera 2009.

\begin{tabular}{|c|l|}
\hline $\begin{array}{l}\text { Número de miembros del hogar menores } \\
\text { de } 16 \text { años }\end{array}$ & $\begin{array}{l}\text { Tiempo medio empleado al día para } \\
\text { necesidades personales }\end{array}$ \\
\hline 0 & 2 horas y 7 minutos \\
\hline 1 & 1 hora y 48 minutos \\
\hline 2 & 1 hora y 39 minutos \\
\hline 3 & 1 hora y 37 minutos \\
\hline Total & 1 hora y 56 minutos \\
\hline
\end{tabular}

Fuente: Datos de Investigación

Esta conclusión es importante, pues confirma la teoría de que una mayor carga familiar influye directamente en una menor dedicación al cuidado personal del individuo/a.

Por último, el modelo construido para explicar la variable tiempo empleado para necesidades personales explica una parte significativa de la variación de la variable en cuestión, concretamente un $69 \%$, lo cual indica que dicho modelo es capaz de determinar un alto porcentaje de la variable dependiente.

El modelo que explica la variación del tiempo empleado para el trabajo doméstico, al incluir todas las variables de control, determina que las únicas variables que influyen en ella son el número de miembros del hogar menores de 16 años y el sexo del encuestado (tabla 6). 
TABLA 6: Variable "tiempo dedicado al trabajo doméstico" según fuente de variación. La Gomera 2009.

\begin{tabular}{|l|l|l|}
\hline Fuente de Variación & F & Significatividad \\
\hline Edad & 0,444 & 0,506 \\
\hline Miembros en el hogar menores de 16 años & 5,781 & 0,017 \\
\hline Sexo & 37,035 & 0,000 \\
\hline Estado Civil & 2,500 & 0,060 \\
\hline Nivel de estudios terminados & 0,614 & 0,689 \\
\hline Interacción Sexo * Estado Civil & 2,260 & 0,082 \\
\hline Interacción Sexo * Nivel de estudios terminados & 0,650 & 0,662 \\
\hline Interacción Estado Civil * Nivel de Estudios terminados & 0,232 & 0,990 \\
\hline Interacción Sexo * Nivel de Estudios Terminados * Estado Civil & 0,198 & 0,986 \\
\hline Varianza explicada por el modelo & $35,40 \%$ & \\
\hline
\end{tabular}

Fuente: Datos de Investigación

Respecto a la relación entre el tiempo empleado al trabajo doméstico y el sexo ya se intuía que existía un fuerte vínculo entre ambas (ver tabla 2). En dicha tabla, se mostraba que las mujeres emplean tres veces y medio más de tiempo en la realización de este tipo de tareas que los hombres. Por otro lado, respecto al número de miembros del hogar menores de 16 años, se observa que a una mayor cantidad de miembros del hogar menores de 16 años, menos tiempo se emplea para la realización del trabajo doméstico (tabla 7).

TABLA 7: Miembros del hogar menores de 16 años y tiempo empleado para el trabajo doméstico. La Gomera 2009.

\begin{tabular}{|c|l|}
\hline $\begin{array}{l}\text { Número de miembros del hogar } \\
\text { menores de } 16 \text { años }\end{array}$ & $\begin{array}{l}\text { Tiempo medio empleado al día } \\
\text { para trabajo doméstico }\end{array}$ \\
\hline 0 & 1 hora y 40 minutos \\
\hline 1 & 1 hora y 43 minutos \\
\hline 2 & 1 hora y 38 minutos \\
\hline 3 & 47 minutos \\
\hline Total & 1 hora y 40 minutos \\
\hline
\end{tabular}

Fuente: Datos de Investigación

Esto se podría explicar, en parte, porque al ser más miembros de ese tipo en el hogar es necesario aumentar las fuentes de ingreso, para lo cual la mujer ha de trabajar fuera de casa empleando menos tiempo al trabajo doméstico. Además, tanto la mujer como el hombre habitantes de hogares con una mayor cantidad de miembros menores de 16 años también podrían recibir la ayuda de estos miembros a la hora de realizar este tipo de labores. Por último, es necesario aclarar que el modelo aquí expuesto (tabla 6) explica un $35 \%$ de la variación de la variable dependiente tiempo empleado para el trabajo doméstico, lo cual permite predecir en buena parte cómo variará ésta.

El tiempo que el encuestado dedica al cuidado de la familia está determinado, según el modelo que explica un porcentaje de variación del $18 \%$ de la variable en cuestión, por los indicadores edad, número de miembros del hogar menores de 16 años, estado civil y sexo (ver tabla 8).

Respecto a esta última variable, y tal y como se expuso en la tabla 2, queda demostrado estadísticamente que las mujeres emplean más del doble de tiempo que los hombres en el cuidado de la familia, por lo que efectivamente el sexo del encuestado influye en el tiempo dedicado a estas labores. 
TABLA 8: variable "tiempo dedicado al cuidado de la familia" según fuente de variación. La Gomera 2009.

\begin{tabular}{|l|l|l|}
\hline Fuente de Variación & F & Significatividad \\
\hline Edad & 11,275 & 0,001 \\
\hline Miembros en el hogar menores de 16 años & 4,983 & 0,026 \\
\hline Sexo & 7,276 & 0,007 \\
\hline Estado Civil & 4,527 & 0,004 \\
\hline Nivel de estudios terminados & 1,438 & 0,211 \\
\hline Interacción Sexo * Estado Civil & 0,769 & 0,512 \\
\hline Interacción Sexo * Nivel de estudios terminados & 0,701 & 0,623 \\
\hline Interacción Estado Civil * Nivel de Estudios terminados & 1,008 & 0,434 \\
\hline Interacción Sexo * Nivel de Estudios Terminados * Estado Civil & 0,404 & 0,899 \\
\hline Varianza explicada por el modelo & $18,20 \%$ & \\
\hline
\end{tabular}

Fuente: Datos de Investigación

La edad, al poseer un nivel de significación menor que 0,05 en el modelo, influye también en la variable dependiente tiempo empleado para el cuidado de la familia.

TABLA 9: Edad y tiempo empleado para el cuidado de la familia. La Gomera 2009

\begin{tabular}{|l|l|}
\hline Edad por grupos & $\begin{array}{l}\text { Tiempo medio empleado al día } \\
\text { para el cuidado de la familia }\end{array}$ \\
\hline De 18 a 29 años & 1 hora y 8 minutos \\
\hline De 30 a 44 años & 1 hora y 43 minutos \\
\hline De 45 a 64 años & 1 hora y 3 minutos \\
\hline 65 y más años & 23 minutos \\
\hline Total & 1 hora y 8 minutos \\
\hline
\end{tabular}

Fuente: Datos de Investigación

Así, y tal y como se observa en la tabla 9, tiene sentido afirmar que los que menos tiempo emplean son los mayores de 65 años debido sobretodo a que son el sector de población que recibe dichos cuidados, siendo los individuos de entre 30 y 44 años los que más tiempo dedican.

TABLA 10: Número de miembros del hogar menores de 16 años y tiempo empleado para el cuidado de la familia. La gomera 2009

\begin{tabular}{|c|l|}
\hline $\begin{array}{l}\text { Número de miembros del hogar } \\
\text { menores de } 16 \text { años }\end{array}$ & $\begin{array}{l}\text { Tiempo medio empleado al día para } \\
\text { el cuidado de la familia }\end{array}$ \\
\hline 0 & 38 minutos \\
\hline 1 & 2 horas y 11 minutos \\
\hline 2 & 2 horas \\
\hline 3 & 1 hora y 44 minutos \\
\hline Total & 1 hora y 19 minutos \\
\hline
\end{tabular}

Fuente: Datos de Investigación

La relación entre el número de miembros del hogar menores de 16 años y el tiempo dedicado al cuidado de la familia (tabla 10) determina que los hogares con uno o dos miembros menores de 16 emplean más tiempo que el resto, siendo los hogares sin ningún miembro menor de dicha edad los que menos tiempo emplean.

Este resultado es coherente, pues al no disponer de ningún miembro menor de 16 años, menos tiempo se dedica a la familia. En cambio, cuando son más de dos miembros los menores de 16 años, el tiempo empleado tiende a disminuir, lo cual indica que al aumentar el número de miembros de ese tipo el empleo de tiempo va siendo cada vez menor. 
Por último, el estado civil del encuestado, según el modelo, es otra variable que influye a la hora de determinar el tiempo empleado para el cuidado de la familia (tabla 11). Según la tabla anterior, los solteros y los viudos son los que menos tiempo emplean en el cuidado de la familia, sobretodo debido a la habitual ausencia de cargas familiares en su vida diaria. Sin embargo, los casados/as y divorciados/as son los que más tiempo emplean, pues habitualmente suelen habitar con una familia que necesita de cuidados. Por contra, es llamativo el hecho de que los divorciados/as son los que dedican una mayor cantidad de tiempo al cuidado de la familia.

TABLA 11: Estado civil del encuestado y tiempo empleado para el cuidado de la familia. La Gomera 2009.

\begin{tabular}{|l|l|}
\hline Estado Civil del Encuestado & $\begin{array}{l}\text { Tiempo medio empleado al día para el cuidado } \\
\text { de la familia }\end{array}$ \\
\hline Soltero/a & 38 minutos \\
\hline Casado/a Viviendo en Pareja & 1 hora y 25 minutos \\
\hline Separado/a o Divorciado/a & 1 hora y 34 minutos \\
\hline Viudo & 26 minutos \\
\hline Total & 1 hora y 9 minutos \\
\hline
\end{tabular}

Fuente: Datos de Investigación

En lo que se refiere al mantenimiento del hogar, la única variable que influye en el tiempo empleado para dicha tarea, según el modelo construido, es el estado civil del encuestado (tabla 12).

TABLA 12: Variable "tiempo dedicado al mantenimiento del hogar" según fuente de variación. La Gomera 2009.

\begin{tabular}{|l|l|l|}
\hline Fuente de Variación & F & Significatividad \\
\hline Edad & 1,935 & 0,165 \\
\hline Miembros en el hogar menores de 16 años & 0,058 & 0,809 \\
\hline Sexo & 0,349 & 0,555 \\
\hline Estado Civil & 2,711 & 0,045 \\
\hline Nivel de estudios terminados & 0,902 & 0,480 \\
\hline Interacción Sexo * Estado Civil & 0,441 & 0,724 \\
\hline Interacción Sexo * Nivel de estudios terminados & 0,784 & 0,536 \\
\hline Interacción Estado Civil * Nivel de Estudios terminados & 0,796 & 0,606 \\
\hline Interacción Sexo * Nivel de Estudios Terminados * Estado Civil & 1,029 & 0,411 \\
\hline Varianza explicada por el modelo & $1,20 \%$ & \\
\hline
\end{tabular}

Fuente: Datos de Investigación

La relación entre ambas (tabla 13) es similar a la establecida entre el tiempo empleado para el cuidado de la familia y el estado civil.

TABLA 13: Estado civil y tiempo empleado para el mantenimiento del hogar

\begin{tabular}{|l|l|}
\hline Estado Civil del Encuestado & $\begin{array}{l}\text { Tiempo medio empleado al día } \\
\text { para el mantenimiento del hogar }\end{array}$ \\
\hline Soltero/a & 42 minutos \\
\hline Casado/a Viviendo en Pareja & 1 hora y 10 minutos \\
\hline Separado/a o Divorciado/a & 59 minutos \\
\hline Viudo & 41 minutos \\
\hline Total & 1 hora \\
\hline
\end{tabular}

Fuente: Datos de Investigación 
De esta forma, los solteros y los viudos son los que menos tiempo dedican, siendo los separados/as y casados/as los que más emplean. Sin embargo, el modelo construido por el cual se explica la variación de la variable tiempo dedicado al mantenimiento del hogar no es muy significativo, pues explica sólo un $1,2 \%$ de la varianza de la variable dependiente. Por lo tanto, no es posible realizar inferencias muy ajustadas respecto al modelo establecido para la citada variable.

Respecto al tiempo empleado para la realización de las tareas del hogar fuera del mismo (tabla 14), no existe ninguna variable del modelo expuesto que influya directamente en la variación de la variable dependiente en cuestión.

TABLA 14: Variable "tiempo dedicado a tareas fuera del hogar" según fuente de variación. La Gomera 2009.

\begin{tabular}{|l|l|l|}
\hline Fuente de Variación & F & Significatividad \\
\hline Edad & 0,271 & 0,603 \\
\hline Miembros en el hogar menores de 16 años & 0,588 & 0,444 \\
\hline Sexo & 2,461 & 0,118 \\
\hline Estado Civil & 0,758 & 0,518 \\
\hline Nivel de estudios terminados & 0,063 & 0,997 \\
\hline Interacción Sexo * Estado Civil & 0,465 & 0,707 \\
\hline Interacción Sexo * Nivel de estudios terminados & 0,430 & 0,827 \\
\hline Interacción Estado Civil * Nivel de Estudios terminados & 0,522 & 0,858 \\
\hline Interacción Sexo * Nivel de Estudios Terminados * Estado Civil & 1,109 & 0,357 \\
\hline Varianza explicada por el modelo & $5,50 \%$ & \\
\hline
\end{tabular}

Fuente: Datos de Investigación

En el tiempo empleado para el tiempo libre, y según el modelo donde se incluyeron las variables de control que explica el 11\% de la variación de la variable dependiente (tabla 15), influyen las siguientes variables: número de miembros del hogar menores de 16 años, estado civil, estado civil y nivel de estudios terminados y sexo. La relación entre esta última variable y el tiempo empleado para el ocio fue descrita en la tabla 2, donde se aprecia que los hombres dedican una mayor cantidad de tiempo a esta cuestión que las mujeres.

TABLA 15: Variable "tiempo dedicado al tiempo libre" según fuente de variación. La Gomera 2009.

\begin{tabular}{|l|l|l|}
\hline Fuente de Variación & F & Significatividad \\
\hline Edad & 0,027 & 0,870 \\
\hline Miembros en el hogar menores de 16 años & 5,261 & 0,023 \\
\hline Sexo & 9,038 & 0,003 \\
\hline Estado Civil & 4,017 & 0,008 \\
\hline Nivel de estudios terminados & 0,361 & 0,875 \\
\hline Interacción Sexo * Estado Civil & 1,138 & 0,334 \\
\hline Interacción Sexo * Nivel de estudios terminados & 0,652 & 0,660 \\
\hline Interacción Estado Civil * Nivel de Estudios terminados & 2,207 & 0,022 \\
\hline Interacción Sexo * Nivel de Estudios Terminados * Estado Civil & 0,373 & 0,918 \\
\hline Varianza explicada por el modelo & $11,00 \%$ \\
\hline
\end{tabular}

Fuente: Datos de Investigación

Por otro lado, el modelo también nos advierte de la relación entre el estado civil y el tiempo empleado para el tiempo libre (tabla 16). 
TABLA 16: Estado civil y tiempo empleado para tiempo libre. La Gomera 2009

\begin{tabular}{|l|l|}
\hline Estado Civil del Encuestado & $\begin{array}{l}\text { Tiempo medio empleado al día para el } \\
\text { tiempo libre }\end{array}$ \\
\hline Soltero/a & 1 hora y 50 minutos \\
\hline Casado/a Viviendo en Pareja & 1 hora y 27 minutos \\
\hline Separado/a o Divorciado/a & 1 hora y 38 minutos \\
\hline Viudo & 1 hora y 45 minutos \\
\hline Total & 1 hora y 35 minutos \\
\hline
\end{tabular}

Fuente: Datos de Investigación

Los solteros/as y los viudos/as son los que más tiempo dedican al día en el ocio, debido sobretodo a que disponen de un mayor espacio temporal dedicado al tiempo libre que el resto de los estados civiles. De igual forma, los casados/as y los divorciados/as son los que menos tiempo dedican, normalmente porque a dichas situaciones suele asociarse algún tipo de carga o responsabilidad familiar.

TABLA 17: Miembros del hogar menores de 16 años y tiempo empleado para el tiempo libre. La Gomera 2009.

\begin{tabular}{|c|l|}
\hline $\begin{array}{l}\text { Número de miembros del hogar menores } \\
\text { de } 16 \text { años }\end{array}$ & $\begin{array}{l}\text { Tiempo medio empleado al día para el } \\
\text { tiempo libre }\end{array}$ \\
\hline 0 & 1 hora y 50 minutos \\
\hline 1 & 1 hora y 25 minutos \\
\hline 2 & 1 hora y 20 minutos \\
\hline 3 & 1 hora y 13 minutos \\
\hline Total & 1 hora y 37 minutos \\
\hline
\end{tabular}

Fuente: Datos de Investigación

Respecto a los miembros del hogar menores de 16 años y el tiempo empleado al día para el tiempo libre (tabla 17), a una mayor cantidad de miembros de esa edad le corresponde una menor proporción de tiempo dedicado a dicha cuestión. Este resultado viene dado porque, lógicamente, a más menores de edad viviendo en el hogar va asociada a una menor cantidad de tiempo para el ocio de los progenitores o del resto de la familia debido sobretodo porque se hace necesaria una mayor dedicación a cuidados de la misma.

Según el modelo construido para determinar la variación de la variable dependiente, al interactuar las variables estado civil y nivel de estudios terminados con el tiempo empleado para el ocio (tabla 18), se determina la influencia de esa interacción en la variable de uso del tiempo en cuestión. Según la tabla anterior, los solteros con estudios secundarios y universitarios son los que más tiempo dedican al tiempo libre, siendo las personas sin estudios las que menos. Sin embargo, respecto a los casados/as, los que más tiempo libre tienen son los de Bachillerato mientras que los que menos son los universitarios. En cambio, si una persona está separada es más probable que cuanto mayor sean sus estudios menores la cantidad de empleo de tiempo libre, cosa que ocurre en sentido contrario respecto a la población viuda: cuanto mayor es el nivel de estudios más tiempo libre disponen.

En otro orden de cosas, resulta necesario destacar qué actividades son las más practicadas en lo que al tiempo libre se refiere (tabla 19).

La actividad más realizada es la de caminar/pasear, seguida de ver la televisión y hacer deporte. A éstas le siguen, con un 13,1\% del total, las actividades relacionadas con el sector primario, estar con amigos y la lectura. 
TABLA 18: Estado civil, nivel de estudios y tiempo empleado para el tiempo libre. La Gomera 2009.

\begin{tabular}{|c|c|c|}
\hline $\begin{array}{l}\text { Estado Civil del } \\
\text { Encuestado } \\
\end{array}$ & Nivel de Estudios Terminados & $\begin{array}{l}\text { Tiempo medio empleado al día } \\
\text { para el tiempo libre }\end{array}$ \\
\hline \multirow[t]{6}{*}{ Soltero/a } & Sin Estudios & 1 hora \\
\hline & Primarios (EGB) & 1 hora y 12 minutos \\
\hline & Secundarios (ESO, BUP, Garantía Social) & 2 horas y 20 minutos \\
\hline & Bachillerato y Ciclos formativos & 1 hora y 47 minutos \\
\hline & Universitarios & 2 horas y 20 minutos \\
\hline & Total & 1 hora y 53 minutos \\
\hline \multirow{7}{*}{$\begin{array}{l}\text { Casado/a } \\
\text { Viviendo en } \\
\text { Pareja }\end{array}$} & No sabe leer ni escribir & 1 hora y 15 minutos \\
\hline & Sin Estudios & 1 hora y 32 minutos \\
\hline & Primarios (EGB) & 1 hora y 20 minutos \\
\hline & Secundarios (ESO, BUP, Garantía Social) & 1 hora y 34 minutos \\
\hline & Bachillerato y Ciclos formativos & 1 hora y 35 minutos \\
\hline & Universitarios & 1 hora 11 minutos \\
\hline & Total & 1 hora y 27 minutos \\
\hline \multirow{6}{*}{$\begin{array}{l}\text { Separado/a o } \\
\text { Divorciado/a }\end{array}$} & Sin Estudios & 2 horas \\
\hline & Primarios (EGB) & 1 hora y 47 minutos \\
\hline & Secundarios (ESO, BUP, Garantía Social) & 1 hora y 52 minutos \\
\hline & \begin{tabular}{|l|} 
Bachillerato y Ciclos formativos \\
\end{tabular} & 1 hora y 17 minutos \\
\hline & Universitarios & 1 hora \\
\hline & Total & 1 hora y 38 minutos \\
\hline \multirow[t]{5}{*}{ Viudo } & No sabe leer ni escribir & 1 hora y 20 minutos \\
\hline & \begin{tabular}{|l|} 
Sin Estudios \\
\end{tabular} & 1 hora y 33 minutos \\
\hline & Primarios (EGB) & 2 hora y 35 minutos \\
\hline & Secundarios (ESO, BUP, Garantía Social) & 2 horas \\
\hline & Total & 1 hora y 45 minutos \\
\hline \multirow[t]{7}{*}{ Total } & No sabe leer ni escribir & 1 hora y 18 minutos \\
\hline & \begin{tabular}{|l|} 
Sin Estudios \\
\end{tabular} & 1 hora y 33 minutos \\
\hline & Primarios (EGB) & 1 hora y 25 minutos \\
\hline & Secundarios (ESO, BUP, Garantía Social) & 1 hora y 56 minutos \\
\hline & Bachillerato y Ciclos formativos & 1 hora y 38 minutos \\
\hline & Universitarios & 1 hora y 42 minutos \\
\hline & Total & 1 hora y 36 minutos \\
\hline
\end{tabular}

Fuente: Datos de Investigación

Respecto a las diferencias por sexo, se observa que ciertas actividades están muy feminizadas, como las de leer (casi el triple de mujeres que de hombres lo practican) o la de estar con amigos, aunque esta última en menor intensidad.

TABLA 19: Actividades realizadas en el tiempo libre según el sexo del encuestado. La Gomera 2009.

\begin{tabular}{|l|l|l|l|l|}
\hline Actividad de tiempo libre & & \multicolumn{2}{|l|}{ Sexo del encuestado } & Total \\
\cline { 3 - 5 } & & Hombre & Mujer & \\
\hline Caminar/Pasear & $\%$ del total & $7,10 \%$ & $10,20 \%$ & $17,30 \%$ \\
\hline Ver Televisión & $\%$ del total & $5,40 \%$ & $5,60 \%$ & $10,90 \%$ \\
\hline Hacer deporte & $\%$ del total & $11,90 \%$ & $2,20 \%$ & $14,10 \%$ \\
\hline Agricultura, Ganadería, Pesca, Jardinería & $\%$ del total & $10,20 \%$ & $2,90 \%$ & $13,10 \%$ \\
\hline Estar con amigos & $\%$ del total & $3,40 \%$ & $4,90 \%$ & $8,30 \%$ \\
\hline Leer & $\%$ del total & $1,90 \%$ & $4,90 \%$ & $6,80 \%$ \\
\hline Otras & $\%$ del total & $11,00 \%$ & $18,50 \%$ & $29,50 \%$ \\
\hline Total & $\%$ del total & $50,90 \%$ & $49,10 \%$ & $100,00 \%$ \\
\hline
\end{tabular}

Fuente: Datos de Investigación 
Sin embargo, los hombres tienen más acaparadas las actividades deportivas y las relacionadas con el sector primario (agricultura, ganadería, jardinería y pesca). De esta forma, las actividades que requieren mayor esfuerzo físico son más demandadas por los hombres que por las mujeres, siendo éstas las que practican actividades de corte intelectual como la lectura.

El tiempo dedicado al trabajo remunerado, según el modelo construido para determinar la variación de la variable en cuestión que nos explica un 29,3\% de la varianza (tabla 20), se ve influido por la edad, el estado civil y el nivel de estudios terminados que posee el encuestado.

TABLA 20: Variable "tiempo dedicado al trabajo remunerado" según fuente de variación. La Gomera 2009

\begin{tabular}{|l|l|l|}
\hline Fuente de Variación & F & Significatividad \\
\hline Edad & 4,236 & 0,040 \\
\hline Miembros en el hogar menores de 16 años & 0,479 & 0,489 \\
\hline Sexo & 0,415 & 0,520 \\
\hline Estado Civil & 5,461 & 0,001 \\
\hline Nivel de estudios terminados & 4,334 & 0,001 \\
\hline Interacción Sexo * Estado Civil & 0,251 & 0,861 \\
\hline Interacción Sexo * Nivel de estudios terminados & 0,438 & 0,822 \\
\hline Interacción Estado Civil * Nivel de Estudios terminados & 1,052 & 0,399 \\
\hline Interacción Sexo * Nivel de Estudios Terminados * Estado Civil & 0,544 & 0,800 \\
\hline Varianza explicada por el modelo & $29,30 \%$ \\
\hline
\end{tabular}

Fuente: Datos de Investigación

Según la tabla 21, el nivel de estudios influye en el tiempo empleado al trabajo remunerado de tal forma que a un mayor nivel de estudios más tiempo se emplea en el trabajo remunerado. Esta cuestión se debe a que el porcentaje de población ocupada es mayor en los sectores más cualificados de la sociedad, pues tienen mayores oportunidades de encontrar trabajo que los que tienen una menor o ninguna cualificación.

TABLA 21: Nivel de estudios y tiempo empleado en el trabajo remunerado.

La Gomera 2009.

\begin{tabular}{|l|l|}
\hline Nivel de Estudios Terminados & $\begin{array}{l}\text { Tiempo medio empleado al } \\
\text { día para el trabajo remunerado }\end{array}$ \\
\hline Sin Estudios & 1 hora y 3 minutos \\
\hline Primarios (EGB) & 5 horas y 47 minutos \\
\hline Secundarios (ESO, BUP, Garantía Social) & 5 horas y 59 minutos \\
\hline Bachillerato y Ciclos formativos & 6 horas y 31 minutos \\
\hline Universitarios & 7 horas y 44 minutos \\
\hline Total & 5 horas y 18 minutos \\
\hline
\end{tabular}

Fuente: Datos de Investigación

La edad también influye, según el modelo construido, en el tiempo empleado para el trabajo remunerado (tabla 22). De esta forma, el intervalo de 30 a 44 años es el que más horas invierte en esta cuestión, resultado sobretodo de la necesidad de aumentar los ingresos debido a las cargas familiares y de que suelen tener contratos de trabajo más estables.

Sin embargo, los jóvenes trabajan menos horas (por las razones citadas, pero a la inversa) al igual que los de entre 45 y 64 años. En último lugar se sitúan los mayores de 65 años, los cuales emplean poco tiempo al trabajo remunerado porque la mayoría están jubilados. 
TABLA 22: Edad y tiempo empleado en el trabajo remunerado. La Gomera 2009.

\begin{tabular}{|l|l|}
\hline Edad por grupos & Tiempo medio empleado al día para el trabajo remunerado \\
\hline De 18 a 29 años & 5 horas y 5 minutos \\
\hline De 30 a 44 años & 7 horas y 26 minutos \\
\hline De 45 a 64 años & 6 horas y 37 minutos \\
\hline 65 y más años & 29 minutos \\
\hline Total & 5 horas y 22 minutos \\
\hline
\end{tabular}

Fuente: Datos de Investigación

Siguiendo con las variables que influyen en el tiempo empleado en el trabajo remunerado, encontramos al estado civil del encuestado (tabla 23). Así, los separados/as dedican una mayor cantidad de horas que los solteros/as y los casados/as, los cuales emplean un tiempo muy parecido al trabajo remunerado. Por último, los viudos/as, a menudo jubilados/as, lógicamente emplean una menor cantidad de tiempo a esta cuestión.

TABLA 23: Estado civil y tiempo empleado en el trabajo remunerado. La Gomera 2009

\begin{tabular}{|l|l|}
\hline Estado Civil del Encuestado & Tiempo medio empleado al día para el trabajo remunerado \\
\hline Soltero/a & 5 horas y 28 minutos \\
\hline Casado/a Viviendo en Pareja & 5 horas y 44 minutos \\
\hline Separado/a o Divorciado/a & 6 horas y 48 minutos \\
\hline Viudo & 40 minutos \\
\hline Total & 5 horas y 22 minutos \\
\hline
\end{tabular}

Fuente: Datos de Investigación

\section{COMPARATIVA NACIONAL-INSULAR}

En este último bloque de análisis se compararán algunos resultados del estudio realizado en la Isla de la Gomera con el realizado por el Instituto de La Mujer para el resto de España elaborado por De la Fuente Sánchez (2007). Sin embargo, antes de llevar a cabo la comparativa, es necesario realizar algunas consideraciones acerca de diferencias entre ambos estudios:

* El estudio del Instituto de La Mujer realiza una comparativa por años, pues fue realizado los años 1993, 1996, 2001 y 2006, siendo el de la Isla de La Gomera únicamente del año 2009, no pudiendo realizar comparaciones temporales en este último.

* En el capítulo de Usos del Tiempo, se ha eliminado la variable del "tiempo dedicado al estudio", pues ha sido poco significativo para el estudio a nivel nacional. Además, el número de variables de control aplicadas al análisis de las diferencias entre hombres y mujeres se ha reducido con el objetivo de ir concretando un modelo teórico abarcable, pues es la primera investigación de este tipo en la Isla.

* Por último, a lo largo de este artículo se han tratado de forma autónoma los usos del tiempo destinados al "cuidado de la familia", "tareas del hogar fuera del mismo", "mantenimiento del hogar" y "trabajo doméstico". Sin embargo, para la comparativa que se va a realizar, se incluirán bajo un único ítem denominado "Trabajo Doméstico", todo ello para homogeneizar los indicadores insulares y nacionales.

* Del estudio de Instituto de La Mujer se extrajeron los datos de tablas prediseñadas en el propio informe de De la Fuente Sánchez. Así, no se explotaron los datos de la base de datos de dicha investigación referida a España, sino que se utilizaron las tablas realizadas por los autores. 
Tras citar las consideraciones a tener en cuenta a la hora de comparar los resultados del estudio realizado para el conjunto de España y el aplicado en La Gomera, en el presente apartado se mostrarán las diferencias y similitudes de los resultados obtenidos en ambos estudios en algunas de las variables estudiadas. En cuanto a los usos del tiempo, los resultados globales (total hombres y mujeres) difieren en algunos aspectos, tal y como se observa en la tabla 24.

TABLA 24: Medias de usos del tiempo según la variable sexo para España 2007 y La Gomera 2009.

\begin{tabular}{|c|c|c|c|c|c|c|}
\hline & \multicolumn{3}{|c|}{ España 2006} & \multicolumn{3}{|c|}{ La Gomera 2009} \\
\hline & Total & Hombres & Mujeres & Total & Hombres & Mujeres \\
\hline $\begin{array}{l}\text { Tiempo medio dedicado a las } \\
\text { necesidades personales }\end{array}$ & $\begin{array}{l}10 \text { horas } y \\
30 \text { minutos }\end{array}$ & $\begin{array}{l}10 \text { horas } y \\
33 \text { minutos }\end{array}$ & $\begin{array}{l}10 \text { horas y } 27 \\
\text { minutos }\end{array}$ & $\begin{array}{l}1 \text { hora y } 59 \\
\text { minutos }\end{array}$ & $\begin{array}{l}1 \text { hora y } 55 \\
\text { minutos }\end{array}$ & $\begin{array}{l}2 \text { horas y } 2 \\
\text { minutos }\end{array}$ \\
\hline $\begin{array}{l}\text { Tiempo medio dedicado al } \\
\text { trabajo doméstico (incluido } \\
\text { cuidado de la familia, tareas } \\
\text { del hogar fuera del mismo, } \\
\text { mantenimiento) }\end{array}$ & $\begin{array}{l}4 \text { horas y } \\
12 \text { minutos }\end{array}$ & $\begin{array}{l}2 \text { horas y } 20 \\
\text { minutos }\end{array}$ & $\begin{array}{l}5 \text { horas y } 59 \\
\text { minutos }\end{array}$ & $\begin{array}{l}4 \text { horas y } 47 \\
\text { minutos }\end{array}$ & $\begin{array}{l}3 \text { horas y } 9 \\
\text { minutos }\end{array}$ & $\begin{array}{l}6 \text { horas y } 32 \\
\text { minutos }\end{array}$ \\
\hline $\begin{array}{l}\text { Tiempo medio dedicado al } \\
\text { Tiempo Libre }\end{array}$ & $\begin{array}{l}7 \text { horas } y \\
42 \text { minutos }\end{array}$ & $\begin{array}{l}8 \text { horas y } 19 \\
\text { minutos }\end{array}$ & $\begin{array}{llll}7 & \text { horas } & y & 7 \\
\text { minutos } & & \end{array}$ & $\begin{array}{l}1 \text { horas y } 36 \\
\text { minutos }\end{array}$ & $\begin{array}{l}1 \text { hora y } 46 \\
\text { minutos }\end{array}$ & $\begin{array}{l}1 \text { hora y } 24 \\
\text { minutos }\end{array}$ \\
\hline $\begin{array}{l}\text { Tiempo medio dedicado al } \\
\text { Trabajo Remunerado }\end{array}$ & $\begin{array}{ll}3 & \text { horas } y \\
28 & \text { minutos }\end{array}$ & $\begin{array}{l}4 \text { horas y } 28 \\
\text { minutos }\end{array}$ & $\begin{array}{l}2 \text { horas y } 31 \\
\text { minutos }\end{array}$ & $\begin{array}{l}5 \text { horas y } 22 \\
\text { minutos }\end{array}$ & \begin{tabular}{|l|}
5 horas y 57 \\
minutos
\end{tabular} & $\begin{array}{l}5 \text { horas y } 22 \\
\text { minutos }\end{array}$ \\
\hline
\end{tabular}

Fuente: Datos de Investigación y De la Fuente Sánchez, 2007.

De esta forma, existen tres esferas donde los usos del tiempo de los españoles y de los habitantes de La Gomera difieren: en la de necesidades personales, en la de trabajo remunerado y en la de tiempo libre. En la primera, la diferencia es de casi 9 horas, siendo el tiempo libre la diferencia de aproximadamente 6 horas. Sin embargo, en cuanto a la de trabajo remunerado, aunque existen resultados algo distantes, se observa que los habitantes de La Gomera trabajan dos horas más al día que el resto. Por otro lado, existen unos resultados similares en ambos estudios en lo que se refiere al tiempo medio dedicado al trabajo doméstico, pues los resultados sólo difieren en algunos minutos.

Resulta de especial relevancia analizar los usos del tiempo según los sexos (tabla 24), comparando los resultados del estudio a nivel nacional con los obtenidos a nivel insular, todo ello con el objetivo de mostrar la feminización, o no, de diferentes tareas relacionadas con la vida cotidiana de las personas. Así, los hombres encuestados en todo el territorio español emplean mucho más tiempo en las necesidades personales y en el tiempo libre y menos en el trabajo remunerado y en el trabajo doméstico. Sin embargo, y respecto a esta última esfera, resulta llamativo que los individuos estudiados en La Gomera empleen mucho más tiempo en las tareas relacionadas con el trabajo doméstico que el resto de los españoles, algo que puede ser un indicador de que La Gomera se encuentra avanzada en el plano de la igualdad. Respecto a las mujeres, los resultados son muy parecidos a los resultados globales de usos del tiempo, si bien existen algunos puntos discordantes. Entre ellos destaca que las mujeres habitantes de La Gomera emplean más de media hora por encima de la media del resto de las españolas en lo que se refiere al trabajo doméstico, si bien los resultados extraídos del estudio a nivel nacional muestran que las mujeres trabajan de forma remunerada la mitad que las gomeras. Si bien anteriormente se indicaba que se ha podido avanzar en cuanto a la igualdad, el hecho de que las mujeres gomeras empleen más tiempo en el trabajo doméstico y más tiempo en el trabajo remunerado puede implicar una ausencia de conciliación de la vida familiar y personal, cuestión importante a la hora de estudiar la desigualdad de género. Sin 
embargo, existe un aspecto positivo de lo anterior: la mujer gomera se ha incorporado al mercado laboral en mayor medida que el resto de las mujeres españolas.

En otro orden de cosas, a la hora de elaborar los diferentes modelos de análisis de las variables de control que influyen en los usos del tiempo, teniendo siempre en cuenta la consideración de que en el estudio de La Gomera se emplearon menos variables de control que en el estudio nacional, los porcentajes de varianza (variación de las variables dependientes usos del tiempo) explicada son relativamente diferentes (tabla 25).

TABLA 25: Varianzas explicadas de los modelos de análisis por las variables de control que influyen en los usos del tiempo. La Gomera 2009 y España 2007

\begin{tabular}{|c|c|c|}
\hline & España 2006 & La Gomera 2009 \\
\hline Tiempo medio dedicado a las necesidades personales & $7,08 \%$ & $69,00 \%$ \\
\hline $\begin{array}{l}\text { Tiempo medio dedicado al trabajo doméstico (incluido cuidado de la } \\
\text { familia, tareas del hogar fuera del mismo, mantenimiento) }\end{array}$ & $24,36 \%$ & $15,08 \% *$ \\
\hline Tiempo medio dedicado al Tiempo Libre & $17,60 \%$ & $11,00 \%$ \\
\hline Tiempo medio dedicado al Trabajo Remunerado & $22,08 \%$ & $29,30 \%$ \\
\hline
\end{tabular}

Fuente: Datos de Investigación y De la Fuente Sánchez, 2007.

Teniendo en cuenta lo anterior, el modelo elaborado para el conjunto del territorio español explica una mayor variación de la variable tiempo medio dedicado al tiempo libre y el tiempo medio dedicado al trabajo doméstico que los modelos realizados a nivel insular.

TABLA 26: Variables significativas, según los modelos de análisis, de las variables de control que influyen en el tiempo. España 2007 y La Gomera 2009.

\begin{tabular}{|c|c|c|}
\hline & España 2006 & La Gomera 2009 \\
\hline $\begin{array}{l}\text { Tiempo medio dedicado a las } \\
\text { necesidades personales }\end{array}$ & Interacción Sexo-Estado Civil. & $\begin{array}{l}\text { Miembros del hogar menores de } \\
16 \text { años }\end{array}$ \\
\hline $\begin{array}{l}\text { Tiempo medio dedicado al } \\
\text { trabajo doméstico (incluido } \\
\text { cuidado de la familia, tareas del } \\
\text { hogar fuera del mismo, } \\
\text { mantenimiento) }\end{array}$ & $\begin{array}{l}\text { Sexo. } \\
\text { Estado Civil. } \\
\text { Miembros del hogar menores de } 16 \text { años } \\
\text { Interacción Sexo-Estado Civil. }\end{array}$ & \begin{tabular}{|l|} 
Sexo \\
Miembros del hogar menores de \\
16 años \\
Edad. \\
Estado Civil.
\end{tabular} \\
\hline $\begin{array}{l}\text { Tiempo medio dedicado al } \\
\text { Tiempo Libre }\end{array}$ & $\begin{array}{l}\text { Sexo. } \\
\text { Edad. } \\
\text { Estado Civil.. }\end{array}$ & \begin{tabular}{|l|} 
Sexo \\
Estado Civil \\
Miembros del hogar menores de \\
16 años \\
Interacción Estado Civil-Nivel de \\
Estudios terminados
\end{tabular} \\
\hline $\begin{array}{l}\text { Tiempo medio dedicado al } \\
\text { Trabajo Remunerado }\end{array}$ & $\begin{array}{l}\text { Sexo } \\
\text { Edad } \\
\text { Estudios Terminados } \\
\text { Estado Civil } \\
\text { Miembros del hogar menores de } 16 \text { años } \\
\text { Interacción Sexo-Estado Civil. }\end{array}$ & $\begin{array}{l}\text { Edad. } \\
\text { Estado Civil. } \\
\text { Nivel de estudios terminados. }\end{array}$ \\
\hline
\end{tabular}

Fuente: De la Fuente (2007) y Datos de la investigación.

Sin embargo, hay que tener en cuenta que dentro del trabajo doméstico se han incluido el resto de esferas relacionadas con él dentro de este estudio (cuidado de la familia, tareas del hogar fuera del mismo, y mantenimiento del hogar, tal y como se indicó anteriormente), con lo cual el resultado de la varianza explicada a nivel insular en cierta manera no es del todo comparable. Los modelos realizados en el ámbito insular explican una mayor varianza en las 
esferas de trabajo remunerado y en la esfera de necesidades personales. Si bien en la primera las diferencias son de unos pocos puntos porcentuales, en cuanto al tiempo medio dedicado a las necesidades personales la diferencia es de más de un $60 \%$, indicando que el modelo elaborado para La Gomera es mucho más útil que el elaborado para el resto del territorio español en lo que se refiere a dicha esfera.

Dentro de los diferentes modelos de análisis de las variables usos del tiempo a través de las diferentes variables de control también encontramos ciertas diferencias entre los estudios aquí comparado (tabla 26).

De esta forma, la variable sexo es más significativa en el estudio elaborado por el Instituto de La Mujer que en el de La Gomera, pues se encuentra influyendo en todas las esferas. Sin embargo, la que más influye en el estudio aplicado a nivel insular es la variable miembros del hogar menores de 16 años, la cual se encuentra en casi todas las esferas.

Las esferas donde más variables influyen a nivel nacional es la de trabajo remunerado y el trabajo doméstico. Sin embargo, en el entorno insular la esfera de tiempo libre y la del trabajo doméstico son las que más variables de control significativas aglutinan.

\section{CONCLUSIONES DE LA INVESTIGACIÓN}

En cuanto al trabajo doméstico, las mujeres emplean casi tres veces y medio más de tiempo que los hombres. También emplean más tiempo en el cuidado de la familia y en la realización de tareas del hogar fuera del mismo, dedicando una menor cantidad para el tiempo libre y el trabajo remunerado, donde los hombres emplean más tiempo. Por lo tanto, el trabajo doméstico, el cuidado de la familia y la realización de tareas del hogar fuera del mismo son actividades todavía feminizadas.

Por otro lado, y según la edad, los hombres emplean menos tiempo que las mujeres en el trabajo doméstico en todos los intervalos de edad, llegando a haber grandes diferencias tanto en los mayores de 65 años como en los jóvenes de 18 a 29. Ese menor empleo del tiempo también ocurre en todos los intervalos de edad cuando se estudió el cuidado de la familia. En el uso del tiempo dedicado al ocio y al mantenimiento del hogar, sólo existen diferencias entre sexos en el intervalo de mayores de 65 años, donde los hombres emplean mucho más tiempo. Por otra parte, en el sector de 30 a 64 años de edad es donde más igualdad en cuanto a usos del tiempo se tiene, existiendo una mayor desigualdad para los menores de $30 \mathrm{y}$ mayores de 65 años.

Al analizar la influencia de diferentes variables de control en los diferentes usos del tiempo, se observan también muchas diferencias. El tiempo dedicado a tareas relacionadas con las necesidades personales depende del número de miembros del hogar menores de 16 años, pues a medida que el número de miembros de 16 o menos años aumenta, menor tiempo se emplea en las necesidades personales. En cuanto al trabajo doméstico, el tiempo empleado depende del número de miembros del hogar menores de 16 años (a mayor cantidad, menos tiempo en el trabajo doméstico) y del sexo (las mujeres triplican a los hombres en este apartado).

A la hora de realizar tareas relacionadas con el cuidado de la familia, las variables que más influyen son el número de miembros menores de 16 años (las personas que residen con uno o dos miembros emplean más tiempo que el resto), la edad (los que más tiempo dedican son los de 30 a 44, siendo los que menos los mayores de 65), el estado civil (los solteros y los viudos dedican menos tiempo que el resto) y el sexo (las mujeres emplean más tiempo). Además, en los que se refiere al mantenimiento del hogar, el estado civil es la única variable que influye a 
la hora de determinar el tiempo empleado en ello, siendo los solteros y los viudos los que menos tiempo dedican.

En cuanto al tiempo empleado en actividades de tiempo libre, éste depende del número de miembros del hogar menores de 16 años (a una mayor cantidad menos tiempo dedica el individuo al ocio), el sexo (las mujeres emplean menos que los hombres), el estado civil (los solteros y los viudos dedican más tiempo que los casados y divorciados) y el nivel de estudios interactuando con el estado civil (los solteros con estudios secundarios y universitarios son los que más tiempo dedican, siendo los separados con estudios avanzados los que menos cantidad de tiempo libre disponen). En este sentido, las actividades más realizadas en el tiempo libre de los encuestados son la de caminar/pasear, seguida de ver la televisión y hacer deporte. Detrás de ellas se sitúan, de mayor a menor porcentaje de encuestados, las relacionadas con el sector primario, estar con amigos y la lectura. Según el sexo, las actividades que requieren mayor esfuerzo físico (sector primario, hacer deporte) son más realizadas por hombres, siendo las de corte más intelectual realizadas por mujeres (lectura).

Respecto al trabajo remunerado, las variables que influyen en el empleo del tiempo son la edad (los jóvenes trabajan menos horas, siendo los de 30 a 44 años los que más trabajan), el estado civil (los separados dedican una mayor cantidad de horas que los solteros y casados) y el nivel de estudios terminados del encuestado (a mayor nivel de estudios más tiempo emplean en el trabajo remunerado).

A la hora de comparar los resultados de España y La Gomera, existen tres esferas donde los usos del tiempo de los españoles y de los habitantes de La Gomera difieren: en la de necesidades personales, en la de trabajo remunerado y en la de tiempo libre. Por otro lado, existen unos resultados similares en ambos estudios en lo que se refiere al tiempo medio dedicado al trabajo doméstico, pues los resultados sólo se distancian en algunos minutos. Los individuos de sexo masculino estudiados en La Gomera emplean más tiempo en las tareas relacionadas con el trabajo doméstico que el resto de los españoles. Mientras, las mujeres gomeras emplean más tiempo en el trabajo doméstico y en el trabajo remunerado que las del resto de España.

Por último, a la hora de analizar la influencia en los diferentes usos del tiempo de algunas variables de control, la variable sexo es más significativa a la hora de observar diferencias en los usos del tiempo en el estudio elaborado por el Instituto de La mujer que en el de La Gomera, pues se encuentra influyendo en todas las esferas de usos del tiempo. Por tanto, cabría pensar que existen menores diferencias entre los hombres y las mujeres en La Gomera que en el resto del territorio Español.

\section{BIBLIOGRAFÍA}

CIRES (1991), Encuesta sobre Uso del Tiempo en España 1991, Centro de Investigaciones sobre la Realidad social.

-- (1996), Encuesta sobre Uso del Tiempo en España 1991, Centro de Investigaciones sobre la Realidad social.

CIS (2000), Estudio 2396, Centro de Investigaciones Sociológicas.

-- (2008), Estudio 2766, Centro de Investigaciones Sociológicas.

CSIC (1990), Encuesta de Nuevas Demandas y necesidades sociales 1990, Consejo Superior de Investigaciones Científicas.

-- (1995), Encuesta de actividades no remuneradas 1995, Consejo Superior de Investigaciones Científicas. 
-- (1998), Encuesta a responsables de Hogar de la Comunidad de Madrid 1998, Consejo Superior de Investigaciones Científicas.

-- (2003), Encuesta sobre uso del tiempo en España 2003, Consejo Superior de Investigaciones Científicas.

-- (2005), Encuesta CSIC sobre trabajo no remunerado en la Comunidad de Madrid 2005, Consejo Superior de Investigaciones Científicas.

-- (2008), Encuesta CSIC sobre trabajo no remunerado en la Comunidad de Madrid 2008, Consejo Superior de Investigaciones Científicas.

INE (2002-2003), Encuesta de empleo del tiempo en España, Instituto Nacional de Estadística.

-- (2009-2010), Encuesta de empleo del tiempo en España, Instituto Nacional de Estadística.

IZQUIERDO, J. et al. (1988), La desigualdad de las mujeres en el uso del tiempo, Madrid, Instituto de La Mujer.

RAMOS, R. (1990), Cronos dividido. Usos del tiempo y desigualdades entre mujeres y hombres en España, Madrid, Instituto de La Mujer.

ALVARO PAGE, M. (1996), Diferencias en el uso del tiempo entre varones y mujeres y otros grupos, REIS, 74, pp. 291-326.

RALDÚA MARTÍN, E. V. (2001), Comparación internacional de los empleos del tiempo de mujeres y hombres, REIS, 94, pp. 105-126.

DURÁN HERAS, M.A. y ROGERO GARCÍA, J. (2009), La investigación sobre usos del tiempo, Cuadernos Metodológicos, Madrid, CIS.

DE LA FUENTE SÁNCHEZ, M. (coord.) (2007), Usos del tiempo, estereotipos, valores y actitudes, Madrid, Instituto de La Mujer, Ministerio de Trabajo y Asuntos Sociales.

\section{Breve currículo:}

\section{Josué Gutiérrez Barroso}

Licenciado en Sociología por la Universidad de La Laguna, 2006 y Diploma de Estudios Avanzados (DEA), marzo 2009. Proyecto de Tesis Doctoral aprobado el 19 de febrero de 2009, titulado La paradoja del cambio generacional en la Isla de La Gomera: consecuencias demográficas, sociales y económicas y adscrito al programa de doctorado Sociedad, Política y Cultura del Departamento de Sociología de la Universidad de La Laguna. Experiencia laboral: cinco años de experiencia (2006-2011) como sociólogo en la Administración Pública (Cabildo de La Gomera y Cabildo Insular de Tenerife). Ha presentado comunicaciones a diversos congresos de Sociología, además de ser ponente en charlas, mesas redondas y otros cursos de índole universitario. 